\title{
Comparison of CE-FDG-PET/CT with CE-FDG-PET/MR in the evaluation of osseous metastases in breast cancer patients
}

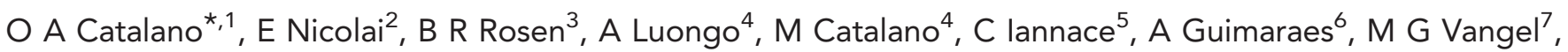
U Mahmood ${ }^{3}$, A Soricelli ${ }^{1}$ and M Salvatore ${ }^{8}$

${ }^{1}$ Department of Radiology, University of Naples Parthenope -SDN IRCCS, Via F. Acton 38, Napoli, Na 80143, Italy; ${ }^{2}$ Department of Nuclear Medicine, SDN IRCCS, Via Gianturco 113, Napoli, Na 80143, Italy; ${ }^{3}$ Department of Radiology, Athinoula A. Martinos Center for Biomedical Imaging, Massachusetts General Hospital, Harvard University Medical School, 149 Thirteenth Street, Charlestown, MA 02129, USA; ${ }^{4}$ Department of Radiology, University of Naples 'Federico II', Naples, NA 80131, Italy; ${ }^{5}$ Department of Surgery, Breast Unit, G. Moscati Hospital, Avellino, AV 83100, Italy; ${ }^{6}$ Department of Radiology, Oregon Health and Sciences University, 3181 Southwest Sam Jackson Park Road, Portland, OR 97239, USA; ${ }^{7}$ Department of Radiology, MGH Biostatistics Center and MGH Martinos Center, Harvard University Medical School, 149 Thirteenth Street, Charlestown, MA 02129, USA and ${ }^{8}$ Department of Radiology and of Nuclear Medicine, SDN IRCCS, Via Gianturco 113, Napoli, Na 80143, Italy

Background: Despite improvements in treatments, metastatic breast cancer remains difficult to cure. Bones constitute the most common site of first-time recurrence, occurring in $40-75 \%$ of cases. Therefore, evaluation for possible osseous metastases is crucial. Technetium $99\left({ }^{99} \mathrm{Tc}\right.$ ) bone scintigraphy and fluorodexossyglucose (FDG) positron emission tomography (PET)-computed tomography (PET-CT) are the most commonly used techniques to assess osseous metastasis. PET magnetic resonance (PET-MR) imaging is an innovative technique still under investigation. We compared the capability of PET-MR to that of same-day PET-CT to assess osseous metastases in patients with breast cancer.

Methods: One hundred and nine patients with breast cancer, who underwent same-day contrast enhanced (CE)-PET-CT and CEPET-MR, were evaluated. CE-PET-CT and CE-PET-MR studies were interpreted by consensus by a radiologist and a nuclear medicine physician. Correlations with prior imaging and follow-up studies were used as the reference standard. Binomial confidence intervals and a $\chi^{2}$ test were used for categorical data, and paired t-test was used for the SUVmax data; a non-informative prior Bayesian approach was used to estimate and compare the specificities.

Results: Osseous metastases affected 25 out 109 patients. Metastases were demonstrated by CE-PET-CT in 22 out of 25 patients $(88 \% \pm 7 \%)$, and by CE-PET-MR in 25 out of 25 patients (100\%). CE-PET-CT revealed 90 osseous metastases and CE-PET-MR revealed 141 osseous metastases $(P<0.001)$. The estimated sensitivity of CE-PET-CT and CE-PET-MR were 0.8519 and 0.9630 , respectively. The estimated specificity for CE-FDG-PET-MR was 0.9884 . The specificity of CE-PET-CT cannot be determined from patient-level data, because CE-PET-CT yielded a false-positive lesion in a patient who also had other, true metastases.

Conclusions: CE-PET-MR detected a higher number of osseous metastases than did same-day CE-PET-CT, and was positive for $12 \%$ of the patients deemed osseous metastasis-negative on the basis of CE-PET-CT.

\footnotetext{
*Correspondence: Professor OA Catalano; E-mail: onofriocatalano@yahoo.it

The paper reports previously unpublished work; however, part of the data has been presented at the RSNA-2014 meeting held in Chicago November/December 2014.
}

Received 7 January 2015; revised 25 February 2015; accepted 27 February 2015; published online 14 April 2015

(C) 2015 Cancer Research UK. All rights reserved 0007-0920/15 
Despite improvements in treatments, metastatic breast cancer remains difficult to cure, with a median survival of 2-3 years and an estimated 10-year survival rate of approximately 10\% (Wood et al, 2005). Among the metastatic organs, bones constitute the most common site of first-time recurrence, occurring in $40-75 \%$ of cases (Wood et al, 2005). Careful evaluation for possible bony metastases is therefore crucial. Technetium $99\left({ }^{99 \mathrm{~m}} \mathrm{Tc}\right) \mathrm{MDP}$ bone scintigraphy and fluorodexossyglucose (FDG) positron emission tomography-computed tomography (PET-CT) are the most commonly used techniques to assess for bony metastasis in breast cancer patients, with reported sensitivity, specificity and accuracy of $67-95.2 \%, 80.9-96 \%$ and $60-80.3 \%$ for ${ }^{99 m} \mathrm{Tc}$ MDP bone scintigraphy and $77.7-96 \%, 88.2-99 \%$ and $92.1-94.1 \%$ for FDGPET-CT (Ohta et al, 2001; Gallowitsch et al, 2003; Hahn et al, 2011; Houssami and Costelloe, 2012; Rong et al, 2013).

However, other techniques have also been employed for the same purpose, including whole-body diffusion weighted magnetic resonance (MR) imaging (WB-DWI) alone or as part of a more comprehensive WB magnetic resonance examination, with reported sensitivity, specificity and accuracy of $86,8,35 \%$ for WB-DWI alone in a selected breast cancer population, with sensitivity and specificity of $33-91 \%$ and $99 \%$ for WB-DWI alone in a mixed population of bony metastases from breast and prostate cancers, and with sensitivity, specificity and accuracy of $88-96.5 \%$, $90-100 \%$ and $91 \%$ for WB magnetic resonance examination (Engelhard et al, 2004; Costelloe et al, 2009; Gutzeit et al, 2010; Heusner et al, 2010). PET magnetic resonance (PET-MR) imaging is an innovative technique whose potential benefits and limitations are still under investigation. In our study, we compared the performance of FDG-PET-CT and FDG-PET-MR in the assessment of bony metastases in a cohort of breast cancer patients.

\section{MATERIALS AND METHODS}

Patient enrolment. This Health Insurance Portability and Accountability Act-compliant retrospective study was approved by the institutional review board. Patients gave written informed consent for study enrolment before undergoing PET-MR. The authors had full control of the data and information submitted for publication. At our institution, as required by the institutional review board and by the Minister of Health, PET-MR can be performed only in patients undergoing same-day PET-CT with the same radioactivity as required for a standalone PET-CT.

Consecutive treated and untreated invasive ductal breast cancer patients who underwent same-day contrast enhanced (CE)-FDGPET-CT and CE-FDG-PET-MR were evaluated for inclusion in this retrospective study. In cases where there were multiple PETCT and PET-MR exams for the same patient, the first retrievable study was evaluated. Inclusion criteria were: (a) invasive ductal breast cancer, (b) $\geq 18$ years of age, (c) same-day CE-FDG-PETCT and CE-FDG-PET-MR, (d) less than 180 min elapsed between FDG injection and PET-MR imaging and (e) availability of comparative imaging including prior exams and/or follow-ups. Exclusion criteria were: (a) pregnancy, (b) blood glucose levels $>140 \mathrm{mg} \mathrm{dl}^{-1}\left(7.77 \mathrm{mmoll}^{-1}\right)$, (c) inadequate PET-CT images, PET-MR images or both, (d) MR contraindications and (e) occurrence of too many lesions to count $(>25)$ to exclude confluent lesions.

Financial support. Although we received no financial support, two authors (BRR and ARG) are consultants for Siemens Healthcare (Erlangen, Germany). Like all other authors, they had access to the data and control of the information.

PET-CT protocols. Patients fasted for at least $6 \mathrm{~h}$ before imaging. Blood glucose levels were assessed before imaging. PET-CT was started a mean of $60 \pm 10 \mathrm{~min}$ after injection of FDG (mean dose \pm s.d., $4.44 \mathrm{MBq}$ per $\mathrm{kg}$ of body weight \pm 1 ; range, $370-400 \mathrm{MBq}$ ). Images were acquired with 64-detector row PETCT scanners (Gemini TF; Philips, Best, the Netherlands) with timeof-flight capabilities. PET-CT was performed at full image quality from mid-thighs to the cranial vault, without and with contrast injection, according to the manufacturer technical protocols. Total acquisition time was $18 \pm 9 \mathrm{~min}$. Technical details of the CE-FDG-PET-CT protocol are outlined in Table 1.

PET-MR protocols. PET-MR was performed with a Biograph mMR imager (Siemens Healthcare, Erlangen, Germany) with a 16-channel head-neck coil and three or four 12-channel body coils depending on patient's height. These coils were combined into a multichannel whole-body coil by using total imaging matrix technology. PET-MR imaging began $125.8 \pm 25.74 \mathrm{~min}$ after FDG injection. Our protocols start with basic co-acquired non$\mathrm{CE}$ (NCE)-MR pulse sequences performed in every patient in conjunction with PET from mid-thighs to the cranial vault. Thereafter, if the patient had not undergone breast surgery, we chose a dedicated 'non-operated breast protocol' that includes dynamic breast sequences, followed by whole-body CE axial and coronal T1-weighted sequences. If breast surgery had already been performed, we opted for an 'operated breast protocol' that includes upper abdominal dynamic sequences as well as whole-body $\mathrm{CE}$ axial and coronal T1-weighted sequences.

The dedicated dynamic breast and upper abdominal sequences were not evaluated, and therefore are not described. The wholebody $\mathrm{CE}$ axial and coronal T1-weighted sequences were evaluated.

Table 1. Technical details of the CE-FDG-PET-CT

\begin{tabular}{|c|c|c|c|c|c|c|c|c|c|}
\hline \multicolumn{3}{|l|}{ CT } & Plane & Area scanned & mAs & kV & $\begin{array}{l}\text { Speed (s per } \\
\text { rotation) }\end{array}$ & Thickness & $\mathrm{FOV}(\mathrm{mm})$ \\
\hline \multicolumn{3}{|c|}{ Attenuation correction } & Axial & Whole body & 80 & 120 & 0.75 & 4.0 & 600 \\
\hline \multicolumn{3}{|c|}{ Diagnostic non-contrast-enhanced } & Axial & Whole body & $250-340$ & 120 & 0.75 & 4.0 & $350-459$ \\
\hline \multicolumn{3}{|c|}{ Diagnostic contrast-enhanced } & Axial & Whole body & $250-340$ & 120 & 0.75 & 4.0 & $350-459$ \\
\hline \multirow[t]{2}{*}{ PET } & BP & $\begin{array}{l}\text { Acquisition } \\
\text { time/BP }\left({ }_{\min }\right)\end{array}$ & \multicolumn{2}{|c|}{$\begin{array}{l}\text { Iterative reconstruc- } \\
\text { tion algorithm }\end{array}$} & Iterations & Subsets & $\begin{array}{l}\text { Axial FOV } \\
(\mathrm{mm})\end{array}$ & $\begin{array}{l}\text { Voxel size } \\
\quad\left(\mathrm{mm}^{3}\right)\end{array}$ & Image grid \\
\hline & $5-7$ & 1.5 & \multicolumn{2}{|c|}{ LMOSEM 3D } & 3 & 33 & 180 & $4 \times 4 \times 4$ & $144 \times 144 \times 144$ \\
\hline \multicolumn{10}{|c|}{$\begin{array}{l}\text { Abbreviations: } \mathrm{BP}=\text { bed position; CE-FDG-PET-CT = contrast enhanced-fluorodexossyglucose-positron emission tomography-computed tomography; FOV }=\text { field of view; } \mathrm{kV}=\mathrm{kilovolt} \text {; } \\
\mathrm{LMOSED} 3 \mathrm{D}=\text { three-dimensional list mode ordered subset expectation maximisation; mAs= milliampere second. PET data and CT attenuation scans were acquired during shallow free } \\
\text { breathing. PET data underwent automatic attenuation correction using attenuation maps generated from attenuation correction CT. Diagnostic non-contrast-enhanced and diagnostic contrast- } \\
\text { enhanced scans were acquired during shallow free breathing for the head, neck, pelvis and upper thighs; they were acquired during breath-hold in expiration for the chest and upper abdomen. } \\
\text { All patients underwent contrast injection. lodine-based contrast medium lomeprol (lopamiro } 370 \text {, Bracco Imaging S.p.A., Milan, Italy) was injected intravenously with a power injector (Empower } \\
\text { CTA, Acist Medical Solutions, Eden Prairie, MN, USA) at } 2 \mathrm{ml} \mathrm{s}^{-1} \text {, at a fixed dose of } 80 \mathrm{ml} \text { for patients weighing }<80 \mathrm{~kg} \text { and at } 100 \mathrm{ml} \text { for those weighing } \geq 80 \mathrm{~kg} \text {; scans were started at the end of } \\
\text { contrast injection. }\end{array}$} \\
\hline
\end{tabular}


Table 2. Technical details of the CE-FDG-PET-MR

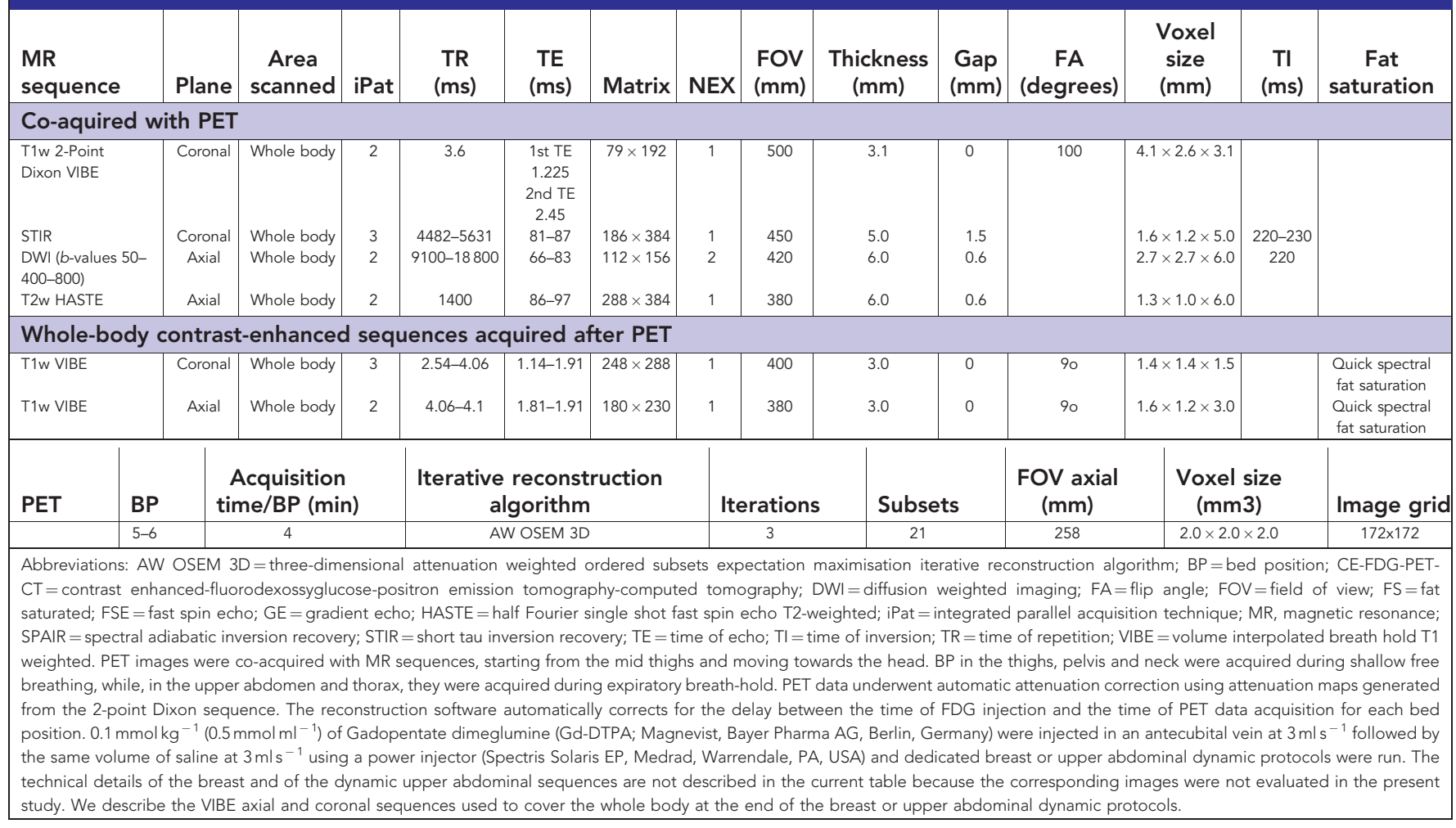

The relevant technical details of the NCE and CE PET-MR acquisitions, which were performed in accordance with the manufacturer technical protocols, are reported in Table 2. Accordingly, in our patient population, only the basic NCEFDG-PET-MR sequences, including the DWI (axial T2-weighted HASTE, axial PET, axial fused HASTE-PET, axial b-800 DWI, ADC map, coronal STIR, coronal T1 in-phase and out-of-phase Dixon) and the whole-body $\mathrm{CE}$ axial and coronal T1-weighted sequences were analysed. Mean total time for the PET-MR examination was $100.97 \pm 21.43$ min. Mean total time for PETMR was $107.87 \pm 18.92 \mathrm{~min}$ in the case of dedicated breast dynamic sequences; otherwise it lasted $94.07 \pm 25.45 \mathrm{~min}$. All the patients were able to tolerate well the whole study.

Image registration and fusion. To be consistent with the manufacturers' indications and with the typical clinical scenarios, the hybrid studies were assessed with the workstations recommended by each of the manufacturers. Therefore, PET-CT images were co-registered, fused by and evaluated at a dedicated workstation (Extended Brilliance Workstation; Philips) and a picture archiving and communication system (IDS7; Sectra, Linkoping, Sweden); whereas PET-MR images were fused by and evaluated at a dedicated workstation (Syngo.via; Siemens Healthcare, Erlangen, Germany) and the aforementioned picture archiving and communication system.

Image evaluation. Two radiology residents (AL and $\mathrm{MC}$ ) searched our breast cancer database and selected patients who satisfied the enrolment and exclusion criteria. For each case, they uploaded the PET-CT or PET-MR images at random. The images were then evaluated by consensus of two readers (a radiologist $(\mathrm{OAC})$ and a nuclear medicine physician (EN) with 14 and 26 years of experience, respectively).

Each hybrid study was evaluated as a whole. When assessing the PET-CT images, the readers simultaneously evaluated the CT images in bone and soft tissue windows, the PET images and the fused PET-CT images. The readers were allowed to modify the window settings as needed. If the readers were assessing the PETMR images, they simultaneously evaluated the axial T2-weighted HASTE, axial PET, axial fused HASTE-PET, axial b-800 DWI, ADC map, coronal STIR, coronal T1 in-phase and out-of-phase Dixon, and axial and coronal post-contrast T1-weighted VIBE. A separate evaluation of whole-body b-800 DWI (WB-DWI) to be compared with PET-CT was also performed.

The readers evaluated only one type of study at a time, either the PET-CT or PET-MR or WB-DWI, for each patient; the remainder of the study was analysed at least 6 weeks later to reduce recall bias. After completion of the PET-CT and PET-MR evaluation, the two readers compared the PET-CT and the PET-MR and the WB-DWI findings and correlation with prior and/or follow-up studies was performed. Readers were aware only of the clinical history of breast cancer. They specifically looked for:

1- Presence or absence of bony metastasis

2- Number of metastases

3- Location (appendicular skeleton, vertebrae, pelvic bones, skull, ribs, sternum)

4- Imaging appearance (lytic, sclerotic, mixed lytic and sclerotic and permeative)

5- Size

6- SUVmax of the five most FDG-avid metastases in each patient.

For both PET-CT and PET-MR imaging, accepted published imaging criteria were used to evaluate for metastasis. Specifically, the following criteria were considered consistent with malignancy: on $\mathrm{CT}$, the occurrence of lytic, sclerotic, mixed sclerotic-lytic changes and/or bone changes with associated soft tissue abnormalities; on MR, focal or diffuse bone marrow intermediate or high signal intensity (SI) on T2-weighted images, STIR, DWI, associated with low SI compared with muscles on T1-weighted sequences, or focal or diffuse areas of persistent low SI on all sequences, 'bull's eye', 'halo sign', extra-osseous tumour infiltration, enhancement after Gadolinium administration, SI changes extending into the 
pedicles and bulging of the posterior and/or anterior margin of the vertebral bodies, ADC values $<1.2 \times 10^{-3} \mathrm{~mm}^{2} \mathrm{~s}^{-1}$; on PET, a focally increased FDG-uptake not associated with significant signs of infection, trauma, degenerative processes, and in equivocal cases an SUV $>2.5$. For WB-DWI only, areas of focal, multifocal or diffuse, irregular high SI within background suppressed bone marrow SI was considered consistent with malignancy.

The following criteria were considered consistent with benignity: on CT location along vertebral endplates, posterior aspect of the spinous process, and along the facets joint, on MR high SI on T1-weighted sequences, location along degenerative changes and/ or along joints, drop of at least a $20 \%$ of SI on out of phase T1-weighted sequences relative to in phase T1-weighted sequences, absence of enhancement, and finally on PET absence of focally increased FDG-uptake (Rybak and Rosenthal, 2001; Schmidt et al, 2007; Kim et al, 2011; Messiou et al, 2011; Padhani and Gogbashian, 2011).

The classification of the imaging appearance of the lesions was mainly derived from CT, in particular, in the case of lytic, sclerotic and mixed lytic and sclerotic; whereas it was mainly derived from $\mathrm{MR}$ in the case of permeative lesions. In the case of discordances between PET-CT and PET-MR, the referring oncologist was consulted to find out if they had impacted on patient's management.

Reference standard. Correlations with prior imaging and followup studies were used as the reference standard. For these patients, we used either appearance of a new bony lesion that satisfied imaging criteria for malignancy and that was absent in the prior studies, increased ${ }^{99} \mathrm{Tc}$ uptake, increased lesion size or increased FDG uptake, or decreased size and decreased FDG uptake after chemotherapy or radiation therapy as confirmation criteria. A case was considered negative when no lesions were identified on both PET-CT and PET-MR and the follow-ups did not disclose any detectable lesions (follow-up length range 347-621 days).

Statistical methods. Results are stated as means plus or minus one standard deviation. Except as noted below, binomial confidence intervals (using the normal approximation) and a $\chi^{2}$ test were used for categorical data, and a paired $t$-test was used for the SUVmax data. We used a non-informative prior Bayesian approach (Box and Tiao, 1973) to estimate and compare the sensitivities of PETMR and PET-CT. We selected this approach, in part, because PETMR was 'perfect' for detecting metastases in all patients who had them, with no false positives. For this situation, the more typical frequentist approach to data analysis breaks down. We assumed binomial likelihoods for the probability of detecting bony metastases, with uniform priors on the binomial parameters. For patients who had metastases, the parameter of the binomial distribution is the sensitivity. For patients who did not have metastases, the binomial parameter is one minus the specificity. We summarised the results with posterior means and equal-tail 95\% credible intervals. We also estimated the probability of PETMR having higher sensitivity than PET-CT using numerical integrations involving the posterior distributions of the binomial parameters.

\section{RESULTS}

A total of 168 breast cancer patients underwent same-day PET-CT and PET-MR between 1 March 2013 and 28 February 2014. In all, 59 patients were excluded: 2 because of severe ferromagnetic MR artefacts due to the breast tissue expanders that partially obscured the lower chest and upper abdomen, 49 due to absence of prior and/or follow-up imaging and 8 because of innumerable bony metastases. Our final study population comprised 109 patients (all women; mean age $=58.08 \pm 10.74$ years). Of the 109 breast cancer patients who satisfied the inclusion criteria, 51 patients, not having undergone breast surgery, underwent dynamic evaluation of the breast during the PET-MR study, meanwhile 58 patients, because of previous breast surgery, underwent dynamic liver assessment during their PET-MR study.

In all, 23 patients had only prior imaging ( 23 out of 23 patients had ${ }^{99} \mathrm{Tc}$ uptake, 23 out of 23 had CT, 1 out of 23 had MR, 20 out of 23 had PET-CT) acquired (mean \pm s.d.) $1354 \pm 903.11$ days, range 354-3278 days, before. A total of 27 patients had only follow-ups (20 out of 27 patients had ${ }^{99} \mathrm{Tc}$ uptake, 27 out of 27 had CT, 3 out of 27 had MR, 23 out of 27 had PET-CT, 23 out of 27 had PET-MR) acquired 449.14 \pm 88.75 days, range 347-630 days, after; and 59 patients had both prior imaging ( 47 out of 59 patients had ${ }^{99}$ Tc uptake, 59 out of 59 had CT, 8 out of 59 had MR, 49 out of 59 had PET-CT) performed $1578.43 \pm 1003.49$ days, range 3943167 days, before as well as follow-ups acquired $516.94 \pm 88.68$ days, range 358-621 days, after the imaging study under analysis (32 out of 59 patients had ${ }^{99} \mathrm{Tc}$ uptake, 59 out of 59 had CT, 2 out of 59 had MR, 59 out of 59 had PET-CT, 59 out of 59 had PET$\mathrm{MR})$. Bony metastases were found in 25 patients ( $23 \pm 4 \%$ ): 9 out of 25 for staging of non-operated breast cancer and 16 out of 25 for restaging of operated breast cancer.

All PET-MR examinations were performed after PET-CT examinations and within $180 \mathrm{~min}$ after FDG injection (mean, $125.8 \pm 25.74 \mathrm{~min})$. Overall, bony metastases were detected by PET-CT in 22 out of $25(88 \pm 7 \%)$ patients and by PET-MR in 25 out of $25(100 \%)$ patients. All lesions detected by both PET-CT and PET-MR were true malignancies, except for one case for which the only candidate lesion detected by PET-CT was actually benign and for two other cases with metastases for which PET-CT detected no lesions. The following results are for true-positive detections only; hence for both PET-CT and PET-MR, the number of cases is 25 , but for 3 cases the number of PET-CT lesions is zero.

In a patient-to-patient comparison, PET-CT and PET-MR findings were concordant in detecting bony metastases in 22 out of $25(88 \pm 7 \%)$ patients, and discordant in 3 out of $25(12 \pm 7 \%)$ patients. With respect to the discordant cases, bony metastases were found with PET-MR but not with PET-CT in 3 out of $25(12 \pm 7 \%)$ patients; moreover, in one patient, a benign area of sclerosis was misinterpreted as metastasis on the CT part of the PET-CT. In a lesion-to-lesion comparison, PET-CT detected 90 bony metastases: 51 out of $90(57 \pm 5 \%)$ on both PET and CT, 37 out of $90(41 \pm 5 \%)$ only on PET without corresponding anatomic correlates and 2 out of $90(2 \pm 2 \%)$ only on CT. Of the 53 metastases visible on CT, 41 were lytic, 9 sclerotic and 3 mixed. One sclerotic and one lytic metastasis were visible only on CT and missed on PET not being FDG avid at the time of the study.

PET-MR detected 141 bony metastases: 85 out of $141(60 \pm 4 \%)$ both on PET and MR, 5 out of $141(4 \pm 2 \%)$ on PET only and 51 out of $141(36 \pm 4 \%)$ on MR only. Overall, MR showed 136 out of $141(96 \pm 2 \%)$ metastases (56 lytic, 9 sclerotic, 3 mixed and 68 permeative). Significantly more lesions were detected using PETMR than with PET-CT $(P<0.001)$. Within the 25 patients with bony metastases, WB-DWI detected 131 bony lesions: 117 true positive metastases (in 24 patients), 14 false positive metastases (in 8 patients), 19 false negative metastases (in 9 patients, 1 of which was deemed free from his single bony metastasis based on WB-DWI).

When we further evaluated the data subdivided according to CT alone, PET-CT, PET-MR, MR and WB-DWI, we found that more lesions were detected by PET-CT than by CT alone and by PETMR than by MR alone for the 22 cases, for which both PET-CT and PET-MR had true-positive results $(88 \pm 6 \%)$. For each of the remaining three cases, a single lesion was detected by both PET-MR and by MR alone, and no lesions were detected correctly by PET-CT. Based on 22 cases, the mean number of additional lesions detected by PETCT as compared with CT alone was 
Table 3. Locations of bone metastases on CE-FDG-PET-CT vs CE-FDG-PET-MR

\begin{tabular}{|c|c|c|}
\hline & CE-FDG-PET-CT & CE-FDG-PET-MR \\
\hline Appendicular skeleton & $2 / 90(2.22 \%)$ & 8/141 (5.67\%) \\
\hline Vertebrae & 29/90 (32.22\%) & $38 / 141$ (26.95\%) \\
\hline Pelvic bones & 35/90 (38.89\%) & $60 / 141(42.55 \%)$ \\
\hline Ribs & $6 / 90(6.67 \%)$ & 9/141 (6.38\%) \\
\hline Sternum & 12/90 (13.33\%) & 18/141 (12.76\%) \\
\hline Skull & $6 / 90(6.67 \%)$ & $8 / 141(5.67 \%)$ \\
\hline \multicolumn{3}{|c|}{$\begin{array}{l}\text { Abbreviations: CE-FDG-PET-CT = contrast enhanced-fluorodexossyglucose-positron emis- } \\
\text { sion tomography-computed tomography; } M R=\text { magnetic resonance. No statistically } \\
\text { significant differences were found between locations of bone metastases on CE-FDG- } \\
\text { PET-CT vs CE-FDG-PET-MR. Most of the bone metastases affected the spine and pelvic } \\
\text { bones. }\end{array}$} \\
\hline
\end{tabular}

$4.0 \pm 4.8$, and the mean number of additional lesions detected by PET-MR as compared with MR alone was $3.6 \pm 4.7$. More lesions were detected by MR alone than by CT alone in 14 cases (56 $\pm 10 \%)$, and the number of additional lesions detected by MR alone was $3.5 \pm 4.5$. Both MR alone and CT alone detected no lesions for the remaining 11 cases.

We subsequently compared true-positive PET-CT, PET-MR and DWI lesion counts. There was one patient with no lesions detected by DWI (and one lesions detected by each of PET-MR and PET-CT), and eight cases with some false-positives DWI lesions $(32 \pm 9 \%)$. More lesions were detected by PET-CT than by DWI for 4 cases $(16 \pm 7 \%)$, with the same number of lesions (all non-zero) detected for 11 cases $(44 \pm 10 \%)$. More lesions were detected by PET-MR than by DWI for 9 cases $(36 \pm 10 \%)$, with the same number of lesions detected for $16(64 \pm 10 \%)$ cases. Lesion locations on PET-CT and on PET-MR are described in Table 3. The locations did not differ significantly between the two modalities $(P=0.83)$. Lesion size was $17.69 \pm 12.64 \mathrm{~mm}$, range 5-54 mm, for all the metastases visible on CT, $13.17 \pm 10.73 \mathrm{~mm}$, range $5-54 \mathrm{~mm}$, for all those visible on $\mathrm{MR}$ and $12.94 \pm 4.66 \mathrm{~mm}$, range $6-28 \mathrm{~mm}$, for those visible only on MR and not visible on CT. The size did not differ significantly between PET-CT and PETMR $(P=1.77)$. SUVmax for PET-CT was $5.00 \pm 3.14$, SUVmax for PET-MR was $5.95 \pm 4.14$, and the difference in means was $-1.48 \pm 2.37$. This difference in means is highly significant $(P<0.00001)$.

For PET-MR, at least some of the metastases were detected for all 25 patients with bony metastases, whereas there were no falsepositives for any of the 84 patients without bony metastases. For PET-CT, at least some of the metastases were detected for 22 of the 25 patients with bony metastases. One of the patients with bony metastases was positive for benign tissue only; we consider this patient as a false-negative for the purpose of sensitivity calculation.

We estimate the sensitivity of PET-CT and PET-MR, respectively, to be 0.8519 with $95 \%$ credible interval $(0.6985,0.9564)$ and 0.9630 with $95 \%$ credible interval $(0.8677,0.9990)$. We estimate the probability that PET-MR has higher sensitivity than PET-CT for detecting bony metastases to be 0.95 . The specificity for PET-MR is estimated to be 0.9884 with $95 \%$ credible interval $(0.9575,0.9997)$. The specificity of PET-CT cannot be determined from patient-level data, because CE-FDG-PET-CT yielded a false-positive in a patient who also had metastases. In the 22 out of 25 patients whose PETCT was positive for bony metastases, no change in the management was induced by detecting more metastases on the same-day PET-MR imaging study. However, in the 3 out of 25 patients, who had already undergone treatments in the past and were considered disease free at the time of their PET-MR, the demonstration on PET-MR only of bony metastases prompted start of radiation therapy and change in hormone therapy in 2, and initiation of chemotherapy in 1 .

\section{DISCUSSION}

We describe our early experience with PET-MR in the detection of bony metastases, based upon a heterogeneous population of breast cancer patients referred for PET-CT. PET-CT is considered pivotal in oncologic management and has been shown to provide more accurate TNM staging than CT or PET alone in several cancers (Antoch et al, 2003; Lardinois et al, 2003; Johnson and Branstetter, 2014). Although PET-CT is increasingly utilised, PET-MR for breast cancer staging has not been evaluated in a clinical setting. PET-CT is not routinely indicated in operable stages I, II and III, or in locally advanced invasive breast cancer stages IIIA, IIIB, IIIC, or even in stage IV disease. The National Comprehensive Cancer Network (NCCN) recommends against using PET-CT in earlystage breast cancer. According to the NCCN guidelines (NCCN guidelines 3.2014), ${ }^{99} \mathrm{Tc}$ scintigraphy or Na-Fluoride-PET-CT are the modality of choice to search for bony metastases in breast cancer patients before treatment, when a systemic staging is being contemplated. PET-CT is deemed 'optional' and 'most helpful when standard staging studies are equivocal or suspicious, especially in the settings of locally advanced or metastatic disease.' The same recommendations apply for follow-up during and after treatment. Bone scans can be omitted when PET-CT is positive for bone metastases.

However, PET-CT is limited by the low-intrinsic tissue contrast of CT, which renders difficulty in the detection of subtle lytic or permeative metastases, and by the degree of glycolytic metabolism. FDG-avid neoplasms need to have a sufficient metabolic activity to be detected by PET (Mueller-Lisse et al, 2007; Ozawa et al, 2010; Sharma et al, 2012). The soft tissue and contrast resolution of MR can help detect lesions and delineate anatomy more clearly than CT (von Schulthess and Schlemmer, 2009; Boss et al, 2010). However, lesion size, location, MR spatial resolution and artefacts may hamper MR performance. MR additionally allows 'functional' evaluation such as with DWI, which provides an alternate contrast mechanism that can be used as a whole-body screening technique to search even for lesions $<10 \mathrm{~mm}$ (Nasu et al, 2006; Padhani et al, 2011). In our study, we compared PET-CT with same-day PETMR for evaluation of bony metastases in breast cancer patients of several stages, irrespective of NCCN guidelines.

PET-MR showed bony metastases in more patients (25 on PETMR and 22 on PET-CT) and overall more lesions (141 metastases on PET-MR and 90 on PET-CT) than PET-CT. In our population, we found PET-MR potentially able to overcome some of the limitations of PET-CT and MR alone (Figures 1-5). According to our experience, and in agreement with data from other studies, PET-MR might be more useful than PET-CT in the evaluation of bony lesions (Catalano et al, 2013). On PET-MR, the concordance of increased FDG uptake with high SI on STIR, lack of a drop in SI on out-of-phase Dixon images and bright contrast enhancement were all suggestive of malignancy (Taoka et al, 2001; Hwang, 2008; Costelloe et al, 2013).

PET and MR can work synergistically to increase the overall diagnostic yield. Not only can MR provide an anatomic correlate for FDG-avid lesions without a definite corresponding CT alteration (Figure 1), it might also be able to detect bony metastases with low FDG uptake that are invisible on PET-CT. These lesions might show high water content on PET-MR, presenting as small lytic or permeative bony lesions. Moreover, the intense enhancement after Gadolinium may increase the diagnostic confidence of the reader (Figure 4; Costelloe et al, 2013). On the other hand, PET can show highly metabolic lesions whose MR correlate might be missed by MR alone, particularly if small (Figure 5). In our population, the fused PET-MR reading helps explain why most of the FDG-avid lesions had a corresponding MR abnormality. 
A

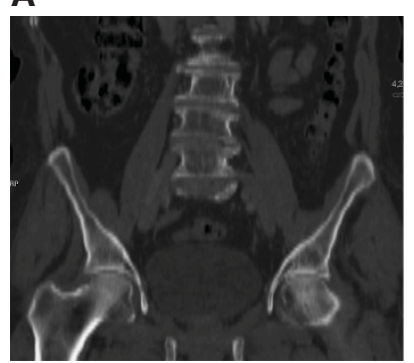

C

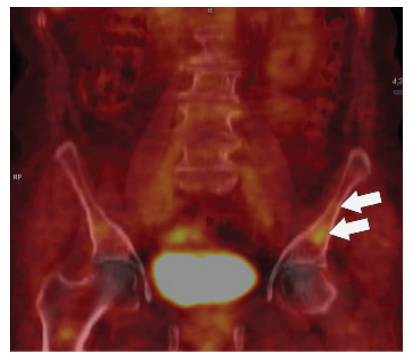

E

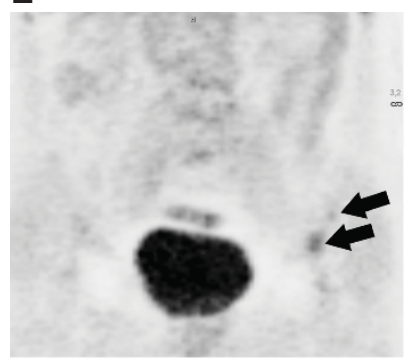

B

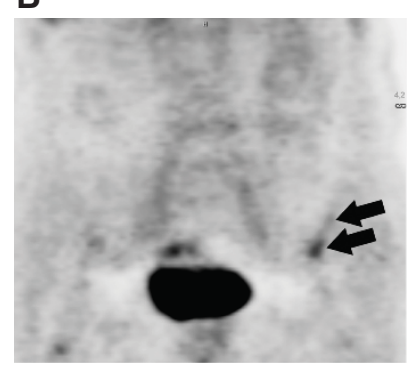

D

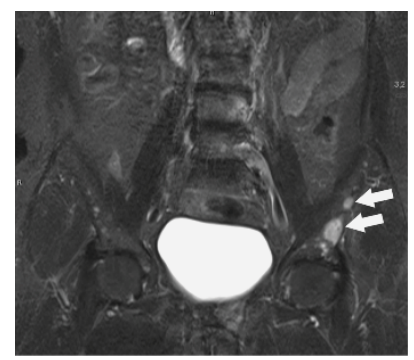

$\mathbf{F}$

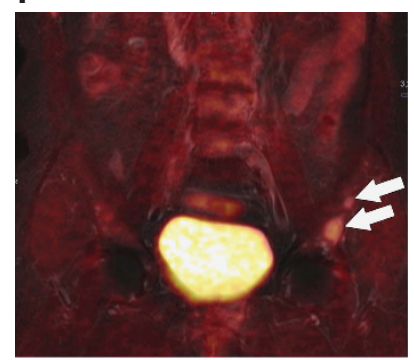

Figure 1. Coronal reformatted CT (A), coronal PET from PET-CT (B), fused coronal PET-CT (C), coronal STIR (D), coronal PET from PET-MR (E), fused coronal PET-MR (F). Two FDG-avid left iliac bony metastases (arrows) can be observed in the PET images obtained from both the PET-CT and the PET-MR scanner. However, no anatomic correlates are visible on $\mathrm{CT}$, whereas they are clearly visible in the STIR image.

In our initial experience, PET-MR is not inferior to PET-CT in detecting sclerotic bony lesions. They may present low FDG uptake, and might be difficult to see on PET; however, they are hypointense on T1-weighted Dixon and hypointense on T2weighted HASTE images. They may present hypointense with adjacent hyperintensity on STIR, and may enhance after Gadolinium. There were no patients who showed bony metastases on PET-CT who did not show bony metastases on PET-MR. In contrast, three patients with no visible bony metastases on PET-CT showed bony metastases on PET-MR. Therefore, we estimate the probability that PET-MR is indeed more sensitive than PET-CT to be 0.95 .

PET-MR detected 141 metastases, whereas PET-CT detected 90 metastases. Of the 51 metastases observed on PET-MR and not on PET-CT, 49 were observed on MR only (5 lytic and 44 permeative) and 2 on both PET and MR (2 permeative). The PET visibility of 2 more metastases on PET-MR compared with the negative FDG uptake on the PET-CT might be related to the delayed acquisition of PET-MR compared with PET-CT with reduced background uptake and increased lesion visibility.

WB-DWI detected 131 bony lesions that were interpreted as metastases; of these 117 were true positives and 14 false positives due to benign bony changes (osteoarthritis, vertebral haemangiomas, osseous infarctions and isolated bone marrow islands) and to magnetic field inhomogeneities due to air interfaces. WB-DWI

A

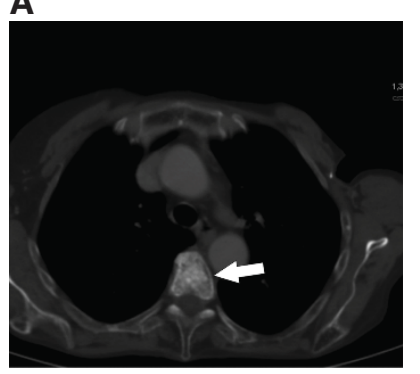

B

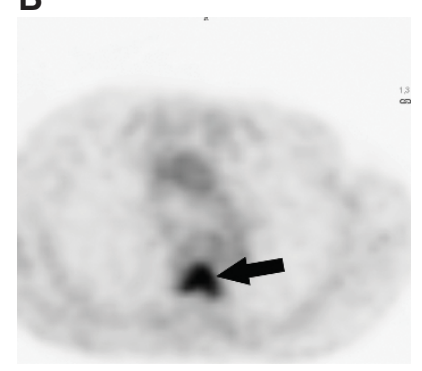

C

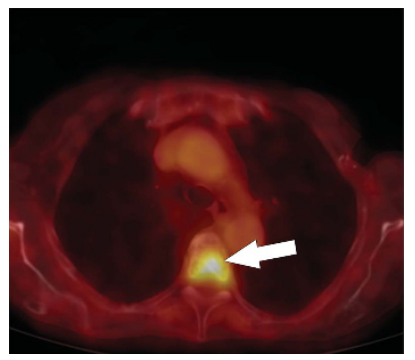

D

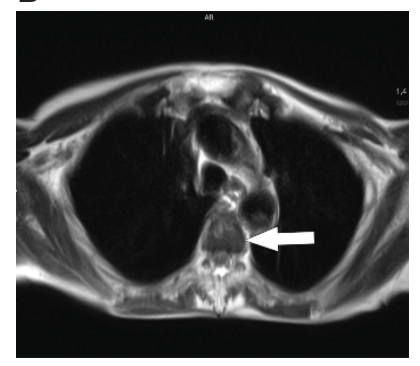

E

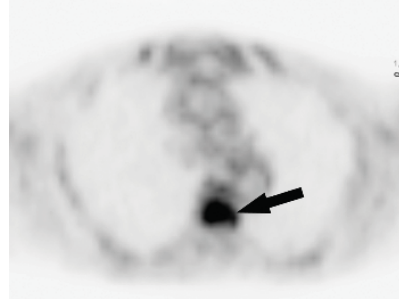

$\mathbf{F}$

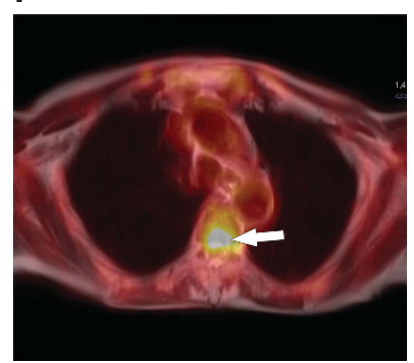

Figure 2. Axial CT (A), axial PET from PET-CT (B), fused axial PET-CT (C), axial HASTE (D), axial PET from PET-MR (E), fused axial PET-MR (F). A large sclerotic FDG-avid vertebral metastasis is identified on CT (arrow) as well as in the corresponding PET image. The reduced signal intensity on same-level HASTE images (D) makes the lesion visible on the MR image as well.

missed 19 metastases visible on the other MR sequences: 9 because of their sclerotic nature and 10 ( 8 lytic plus 2 permeative) due to inappropriate fat suppression at the thoracic inlet/neck region with associated chemical shift artefacts, and due to motion as well as air magnetic susceptibility artefacts. Of these 19 bony metastases not visible on WB-DWI but visible on other MR sequences, 10 were FDG avid (2 sclerotic, 7 lytic and 1 permeative). WB-DWI did not pick up the five metastases visible only on PET and not on MR. The high sensitivity and intermediate to low specificity of WBDWI that we experienced is in agreement with previous studies; to improve WB-DWI performance, it should be interpreted, as we did in the joined evaluation of all our PET-MR sequences, along with conventional MR sequences and with ADC maps, and in the case of PET-MR, with the associated PET data (Wu et al, 2011; Lecouvet et al, 2012). In our experience, the increased sensitivity of WB-DWI has a reduced specificity when compared with PET-CT that makes it less beneficial.

On PET-CT in two patients, no bony lesions were found with potential under-staging; in one other patient, a benign area of sclerosis was misinterpreted as metastases and a permeative bony metastases was not detected. These three patients did not have other metastases at the time of the scanning. In these 3 out of 25 patients, management was directly impacted by PET-MR. The two patients whose PET-CT was read negative underwent radiation therapy on their single bony metastases and change in the 
A

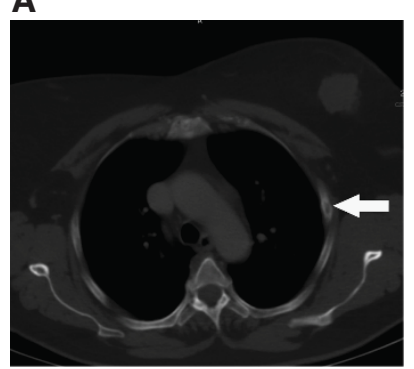

C

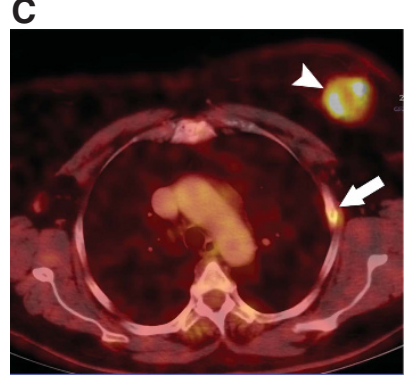

E

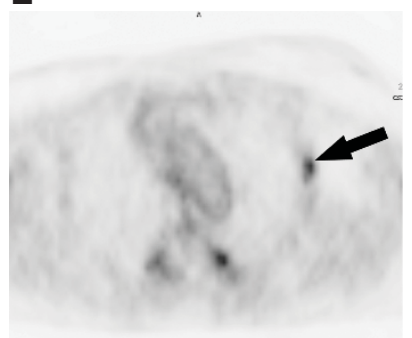

B

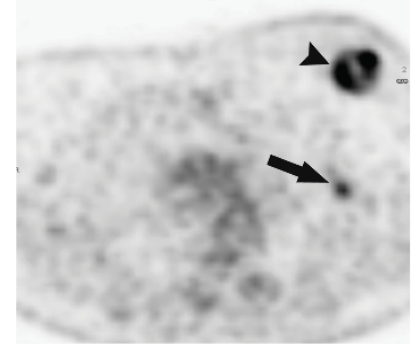

D

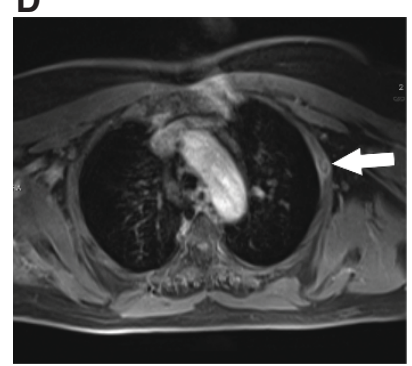

$\mathbf{F}$

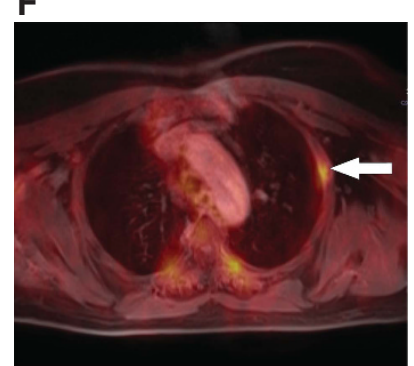

Figure 3. Axial CT (A), axial PET from PET-CT (B), fused axial PET-CT (C), axial contrast-enhanced VIBE (D), axial PET from PET-MR (E), fused axial PET-MR (F). A lytic FDG-avid left rib metastasis, with destruction of the bony marrow and thinning of the cortex, is indicated by arrow in the PET-CT images. The metastasis is also well seen on same-level PET-MR because of the contrast enhancement observed in the VIBE image, as well as to the increased FDG uptake. Please note increased left costo-vertebral junction uptake on PET-MR due to arthritis. The position of the arms along the body during PET-MR acquisition, and beyond the head during PET-CT scanning, explains the concurrent visibility of the primary breast cancer (red arrow) on PET-CT only.

hormonal therapy; in the latter patient, chemotherapy was introduced and she presented progression at the 6-month followup. Our experience is in agreement with that of Souvatsoglou who found, using a different tracer than ours, ${ }^{11} \mathrm{C}$-Choline-PET-MR to be better than ${ }^{11} \mathrm{C}$-Choline-PET-CT in anatomical allocation of bony metastasis from prostate cancer (Souvatzoglou et al, 2013).

When we correlated the SUVmax from FDG-PET-CT with that from PET-MR, differently than in other papers, the SUV from PET-MR $(5.95 \pm 4.14)$ was slightly higher than that from PET-CT $(5 \pm 3.14)$ (Eiber et al, 2014). This discrepancy might be related, at least in part, to the time between the two scans (58.24 $\pm 15.17 \mathrm{~min})$. However, all of the lesions that were FDG avid in the PET-CT study were also avid in the PET-MR study. Two lesions, whose FDG uptake was pathologic with PET-MR being prominent against absent background activity and with SUVmax 2.53 and 2.73, respectively, were undetectable on PET-CT due to similar FDG uptake as adjacent normal bone.

Our approach to PET-MR has been to use both MR and PET to their full potential, and together, similar to the approach by Stolzman for lung nodules, and by others for whole-body oncologic

A

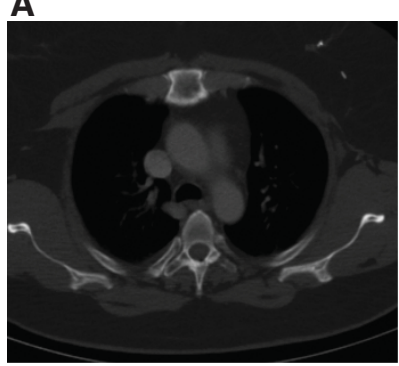

C

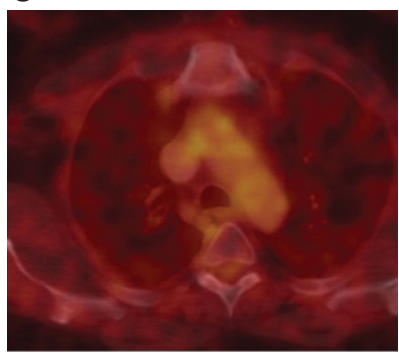

E

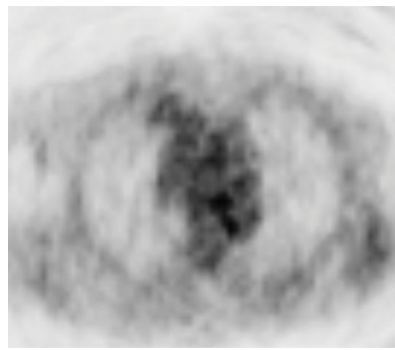

G

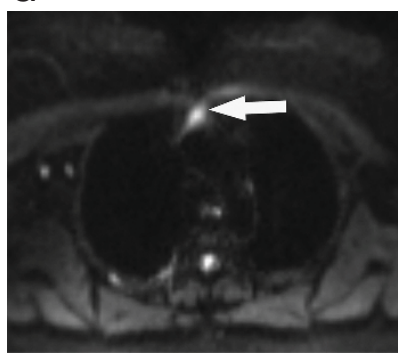

B

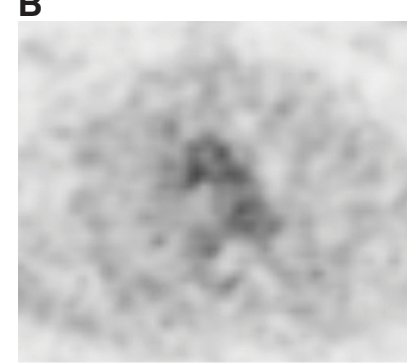

D

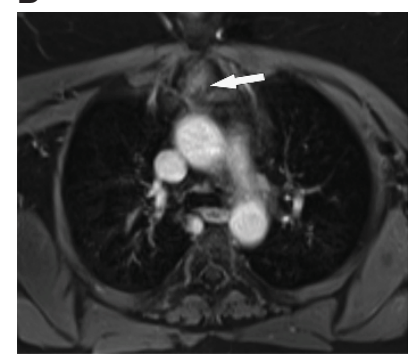

$\mathbf{F}$

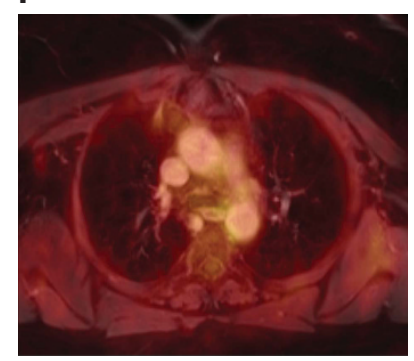

H

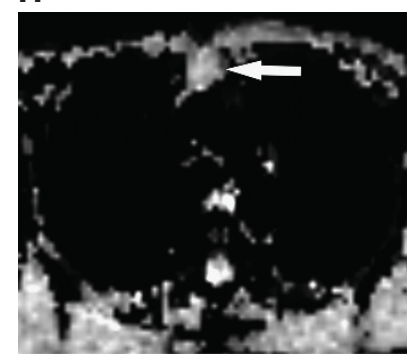

I

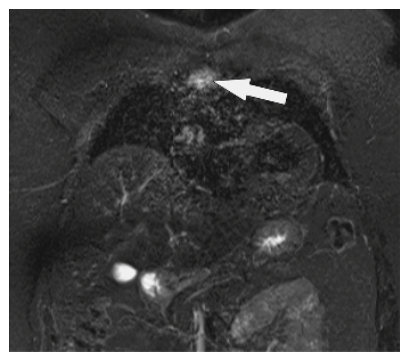

Figure 4. Axial CT (A), axial PET from PET-CT (B), fused axial PET-CT (C), axial contrast-enhanced VIBE (D), axial PET from PET-MR (E), fused axial PET-MR (F), axial 800 b-value DWI (G), axial ADC map image $(\mathrm{H})$ and coronal STIR (I). No lesions are identified on the PETCT examination. However, an area of intense enhancement (arrow in D) with corresponding restricted diffusion $(G, H)$ and increased signal on STIR (I) is appreciated in the manubrium sterni. The lesion, corresponding to a permeative bony metastasis, is devoid of FDG activity. 
A

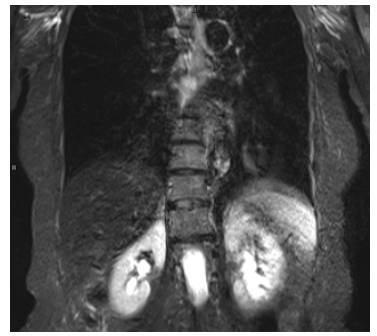

C

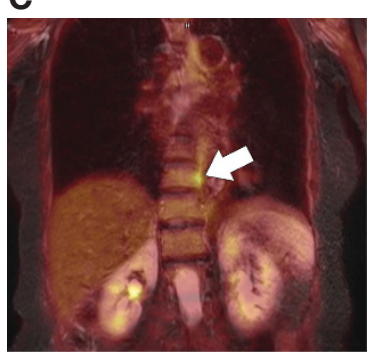

E

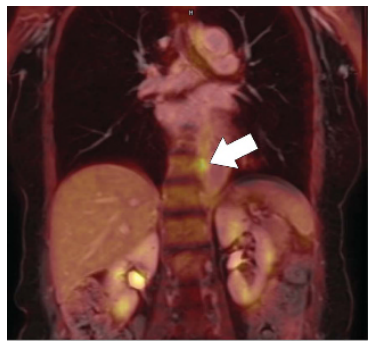

B

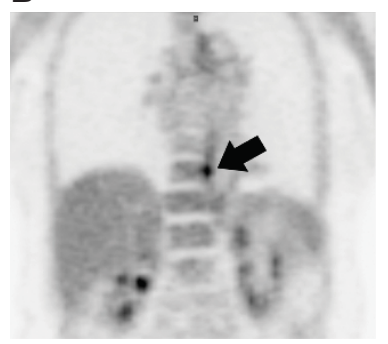

D

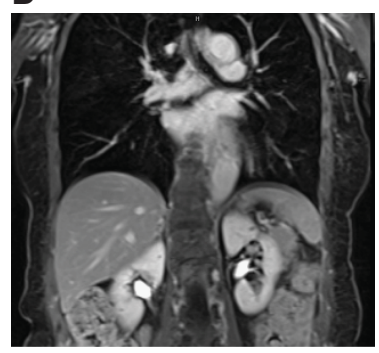

$\mathbf{F}$

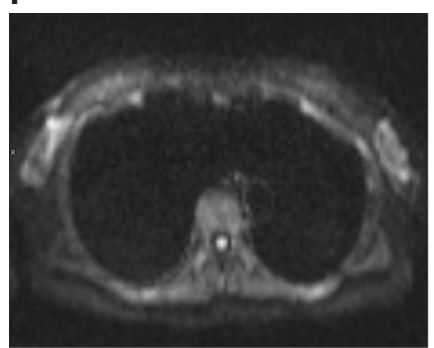

Figure 5. Coronal STIR (A), coronal PET from PET-MR (B), fused coronal PET-STIR (C), coronal contrast-enhanced VIBE (D), coronal fused PET-contrast-enhanced VIBE (E) and axial 800 b-value DWI (F). A focal area of marked FDG uptake, corresponding to a bony metastasis, is identified within the left vertebral body of D10 (arrow in B, C, E). No lesions are identified on the corresponding MR images.

evaluation (Catalano et al, 2013; Stolzmann et al, 2013). Our results are similar to those from Beiderwellen who identified $100 \%$ of bony metastases from different primaries using PET-MR (Beiderwellen et al, 2014). Differently from the approach by Eiber, who used only Dixon and T1-weighted FSE, we used more sequences, reflecting the current clinical MR protocols (Eiber et al, 2010).

Our study has several limitations. First, PET-MR constitutes an innovative technique for which experience and consensus regarding imaging protocols is lacking. Another limitation is the lack of a pathological reference standard, related to the difficulty in performing image-guided bone biopsy for lesions that are invisible on CT. Therefore, in our retrospective study, we relied on prior imaging and/or follow-up for reference standard. Finally, there is a potential selection bias introduced by the retrospective nature of the study and by its being based on referral.

As per recommendations of the European Association of Nuclear Medicine, our Institutional Review Board requested that PET-CT to be acquired $60 \mathrm{~min}$ after FDG administration. Therefore, PET-MR was always performed after PET-CT completion (Boellaard et al, 2010). Although delayed PET acquisition may improve image quality by decreasing background activity, it is unclear whether this improves diagnostic accuracy (Chen et al, 2008; Laffon et al, 2009; Cheng et al, 2013). Our PET-MR protocol includes several sequences aimed to allow a comprehensive wholebody staging as well as a local breast staging, at the cost of a lengthy

examination. Although we are in the process of reducing the duration of the PET-MR study and the number of sequences employed, the aim of the current study was not related to sequence comparison and protocol refinement. Moreover, the evaluation of multiple sequences might account for the absence of false positive cases that might potentially have occurred relying only on DWI or on selected sequences for the diagnosis. Finally, larger studies will be needed to confirm our preliminary results.

\section{CONCLUSIONS}

In the case of bony metastases from breast cancer, PET-MR showed bony metastases not visible on same-day PET-CT in $12 \%$ of the positive patients, impacting on patients' management, and also a higher number of bony metastases (141) than did same-day PET-CT (90).

\section{ACKNOWLEDGEMENTS}

We acknowledge Rosanna Dente for patients gathering and follow-ups, Maria Lepore, MD, for her assistance with pathology, and Gary Boas, PhD, for editing the manuscript.

\section{REFERENCES}

Antoch G, Stattaus J, Nemat AT, Marnitz S, Beyer T, Kuehl H, Bockisch A, Debatin JF, Freudenberg LS (2003) Non-small cell lung cancer: dualmodality PET/CT in preoperative staging. Radiology 229: 526-533.

Beiderwellen K, Huebner M, Heusch P, Grueneisen J, Ruhlmann V, Nensa F, Kuehl H, Umutlu L, Rosenbaum-Krumme S, Lauenstein TC (2014) Whole-body [(1)(8)F]FDG PET/MRI vs. PET/CT in the assessment of bone lesions in oncological patients: initial results. Eur Radiol 24: 20232030.

Boellaard R, O'Doherty MJ, Weber WA, Mottaghy FM, Lonsdale MN, Stroobants SG, Oyen WJ, Kotzerke J, Hoekstra OS, Pruim J, Marsden PK, Tatsch K, Hoekstra CJ, Visser EP, Arends B, Verzijlbergen FJ, Zijlstra JM, Comans EF, Lammertsma AA, Paans AM, Willemsen AT, Beyer T, Bockisch A, SchaeferProkop C, Delbeke D, Baum RP, Chiti A, Krause BJ (2010) FDG PET and PET/CT: EANM procedure guidelines for

tumour PET imaging: version 1.0. Eur J Nucl Med Mol Imaging 37: 181-200.

Boss A, Bisdas S, Kolb A, Hofmann M, Ernemann U, Claussen CD,

Pfannenberg C, Pichler BJ, Reimold M, Stegger L (2010) Hybrid PET/MRI of intracranial masses: initial experiences and comparison to PET/CT. $J$ Nucl Med 51: 1198-1205.

Box GEP, Tiao GC (1973) Bayesian Inference in Statistical Analysis. Addison-Wesley Pub. Co.Reading, Massachusetts..

Catalano OA, Rosen BR, Sahani DV, Hahn PF, Guimaraes AR, Vangel MG, Nicolai E, Soricelli A, Salvatore M (2013) Clinical impact of PET/MR imaging in patients with cancer undergoing same-day PET/CT: initial experience in 134 patients-a hypothesis-generating exploratory study. Radiology 269: 857-869.

Chen YM, Huang G, Sun XG, Liu JJ, Chen T, Shi YP, Wan LR (2008) Optimizing delayed scan time for FDG PET: comparison of the early and late delayed scan. Nucl Med Commun 29: 425-430.

Cheng G, Torigian DA, Zhuang H, Alavi A (2013) When should we recommend use of dual time-point and delayed time-point imaging techniques in FDG PET? Eur J Nucl Med Mol Imaging 40(5): 779-787.

Costelloe CM, Madewell JE, Kundra V, Harrell RK, Bassett Jr. RL, Ma J (2013) Conspicuity of bone metastases on fast Dixon-based multisequence whole-body MRI: clinical utility per sequence. Magn Reson Imaging 31: 669-675.

Costelloe CM, Rohren EM, Madewell JE, Hamaoka T, Theriault RL, Yu TK, Lewis VO, Ma J, Stafford RJ, Tari AM, Hortobagyi GN, Ueno NT (2009) Imaging bone metastases in breast cancer: techniques and recommendations for diagnosis. Lancet Oncol 10: 606-614.

Eiber M, Beer AJ, Holzapfel K, Tauber R, Ganter C, Weirich G, Krause BJ, Rummeny EJ, Gaa J (2010) Preliminary results for characterization of 
pelvic lymph nodes in patients with prostate cancer by diffusion-weighted MR-imaging. Invest Radiol 45: 15-23.

Eiber M, Takei T, Souvatzoglou M, Mayerhoefer ME, Furst S, Gaertner FC, Loeffelbein DJ, Rummeny EJ, Ziegler SI, Schwaiger M, Beer AJ (2014) Performance of whole-body integrated 18F-FDG PET/MR in comparison to PET/CT for evaluation of malignant bone lesions. J Nucl Med 55: 191197.

Engelhard K, Hollenbach HP, Wohlfart K, Von Imhoff E, Fellner FA (2004) Comparison of whole-body MRI with automatic moving table technique and bone scintigraphy for screening for bone metastases in patients with breast cancer. Eur Radiol 14: 99-105.

Gallowitsch HJ, Kresnik E, Gasser J, Kumnig G, Igerc I, Mikosch P, Lind P (2003) F-18 fluorodeoxyglucose positron-emission tomography in the diagnosis of tumor recurrence and metastases in the follow-up of patients with breast carcinoma: a comparison to conventional imaging. Invest Radiol 38: 250-256.

Gutzeit A, Doert A, Froehlich JM, Eckhardt BP, Meili A, Scherr P, Schmid DT, Graf N, Von Weymarn CA, Willemse EM, Binkert CA (2010) Comparison of diffusion-weighted whole body MRI and skeletal scintigraphy for the detection of bone metastases in patients with prostate or breast carcinoma. Skeletal Radiol 39: 333-343.

Hahn S, Heusner T, Kummel S, Koninger A, Nagarajah J, Muller S, Boy C, Forsting M, Bockisch A, Antoch G, Stahl A (2011) Comparison of FDG$\mathrm{PET} / \mathrm{CT}$ and bone scintigraphy for detection of bone metastases in breast cancer. Acta Radiol 52: 1009-1014.

Heusner TA, Kuemmel S, Koeninger A, Hamami ME, Hahn S, Quinsten A, Bockisch A, Forsting M, Lauenstein T, Antoch G, Stahl A (2010) Diagnostic value of diffusion-weighted magnetic resonance imaging (DWI) compared to FDG PET/CT for whole-body breast cancer staging. Eur J Nucl Med Mol Imaging 37: 1077-1086.

Houssami N, Costelloe CM (2012) Imaging bone metastases in breast cancer: evidence on comparative test accuracy. Ann Oncol 23: 834-843.

Hwang S (2008) Imaging of lymphoma of the musculoskeletal system. Radiol Clin North Am 46: 379-396.

Johnson JT, Branstetter BFT (2014) PET/CT in head and neck oncology: State-of-the-art 2013. Laryngoscope 124: 913-915.

Kim SH, Smith SE, Mulligan ME (2011) Hematopoietic tumors and metastases involving bone. Radiol Clin North Am 49: 1163-1183vi.

Laffon E, De Clermont H, Begueret H, Vernejoux JM, Thumerel M, Marthan R, Ducassou D (2009) Assessment of dual-time-point 18F-FDG-PET imaging for pulmonary lesions. Nucl Med Commun 30: 455-461.

Lardinois D, Weder W, Hany TF, Kamel EM, Korom S, Seifert B, von Schulthess GK, Steinert HC (2003) Staging of non-small-cell lung cancer with integrated positron-emission tomography and computed tomography. N Engl J Med 348: 2500-2507.

Lecouvet FE, El Mouedden J, Collette L, Coche E, Danse E, Jamar F, Machiels JP, Vande Berg B, Omoumi P, Tombal B (2012) Can whole-body magnetic resonance imaging with diffusion-weighted imaging replace Tc $99 \mathrm{~m}$ bone scanning and computed tomography for single-step detection of metastases in patients with high-risk prostate cancer? Eur Urol 62: 68-75.

Messiou C, Collins DJ, Morgan VA, Desouza NM (2011) Optimising diffusion weighted MRI for imaging metastatic and myeloma bone disease and assessing reproducibility. Eur Radiol 21: 1713-1718.

Mueller-Lisse UG, Mueller-Lisse UL, Meindl T, Coppenrath E, Degenhart C, Graser A, Scherr M, Reiser MF (2007) Staging of renal cell carcinoma. Eur Radiol 17: 2268-2277.
Nasu K, Kuroki Y, Nawano S, KurokI S, Tsukamoto T, Yamamoto S, Motoori K, Ueda T (2006) Hepatic metastases: diffusion-weighted sensitivityencoding versus SPIO-enhanced MR imaging. Radiology 239: 122-130.

Ohta M, Tokuda Y, Suzuki Y, Kubota M, Makuuchi H, Tajima T, Nasu S, Suzuki Y, Yasuda S, Shohtsu A (2001) Whole body PET for the evaluation of bony metastases in patients with breast cancer: comparison with 99Tcm-MDP bone scintigraphy. Nucl Med Commun 22: 875-879.

Ozawa Y, Hara M, Sakurai K, Nakagawa M, Tamaki T, Nishio M, Shibamoto Y (2010) Diagnostic accuracy of F-18-2-deoxy-fluoro-D-glucose positron emission tomography for $\mathrm{pN} 2$ lymph nodes in patients with lung cancer. Acta Radiologica 51: 150-155.

Padhani AR, Gogbashian A (2011) Bony metastases: assessing response to therapy with whole-body diffusion MRI. Cancer Imaging $11 \mathrm{Spec}$ No A: S129-S145.

Padhani AR, Koh DM, Collins DJ (2011) Whole-body diffusion-weighted MR imaging in cancer: current status and research directions. Radiology 261: $700-718$.

Rong J, Wang S, Ding Q, Yun M, Zheng Z, Ye S (2013) Comparison of 18 FDG PET-CT and bone scintigraphy for detection of bone metastases in breast cancer patients. A meta-analysis. Surg Oncol 22: 86-91.

Rybak LD, Rosenthal DI (2001) Radiological imaging for the diagnosis of bone metastases. Q J Nucl Med 45: 53-64.

Schmidt GP, Schoenberg SO, Schmid R, Stahl R, Tiling R, Becker CR, Reiser MF, Baur-Melnyk A (2007) Screening for bone metastases: whole-body MRI using a 32-channel system versus dual-modality PET-CT. Eur Radiol 17: 939-949.

Sharma P, Kumar R, Singh H, Jeph S, Sharma DN, Bal C, Malhotra A (2012) Carcinoma endometrium: role of 18-FDG PET/CT for detection of suspected recurrence. Clin Nucl Med 37: 649-655.

Souvatzoglou M, Eiber M, Takei T, Furst S, Maurer T, Gaertner F, GEINITZ H, Drzezga A, Ziegler S, Nekolla SG, Rummeny EJ, Schwaiger M, Beer AJ (2013) Comparison of integrated whole-body [C]choline PET/MR with PET/CT in patients with prostate cancer. Eur J Nucl Med Mol Imaging 40(10): 1486-1499.

Stolzmann P, Veit-Haibach P, Chuck N, Rossi C, Frauenfelder T, Alkadhi H, von Schulthess G, Boss A (2013) Detection rate, location, and size of pulmonary nodules in trimodality PET/CT-MR: comparison of low-dose CT and Dixon-based MR imaging. Invest Radiol 48: 241-246.

Taoka T, Mayr NA, Lee HJ, Yuh WT, Simonson TM, Rezai K, Berbaum KS (2001) Factors influencing visualization of vertebral metastases on MR imaging versus bone scintigraphy. AJR Am J Roentgenol 176: 1525-1530. von Schulthess GK, Schlemmer HP (2009) A look ahead: PET/MR versus PET/CT. Eur J Nucl Med Mol Imaging 36(Suppl 1): S3-S9.

Wood WC, Muss HB, Solin LJ, Olopade OI (2005) Cancer, Principles \& Practice of Oncology. Lippincott Williams \& Wilkins: Philadelphia, PA.

Wu LM, Gu HY, Zheng J, Xu X, Lin LH, Deng X, Zhang W, Xu JR (2011) Diagnostic value of whole-body magnetic resonance imaging for bone metastases: a systematic review and meta-analysis. J Magn Reson Imaging 34: $128-135$.

This work is published under the standard license to publish agreement. After 12 months the work will become freely available and the license terms will switch to a Creative Commons AttributionNonCommercial-Share Alike 4.0 Unported License. 